\title{
30th anniversary of Annals of Nuclear Medicine
}

\author{
Seigo Kinuya ${ }^{1}$
}

Published online: 9 December 2015

(C) The Japanese Society of Nuclear Medicine 2015

Dear colleagues,

It is my great pleasure to inform all of you that the Annals of Nuclear Medicine, ANM, celebrates its 30th anniversary this year, having been founded in 1987 under the leadership of Dr. Kinichi Hisada, then-president of the Japanese Society of Nuclear Medicine. The directors of the society at that time determined to eliminate all Japanese words from the journal in order to develop it as an international journal. From that time, ANM has been managed by 11 chief editors including myself. ANM is now indeed one of major global journals in our field.

ANM started with a single issue in 1987, steadily increased the number of issues and now publishes 10 issues containing about 120 articles every year. It was published by a domestic publisher initially, and then by a major international publisher, Springer from 2006. An impact factor was first assigned to ANM for the assessment year of 2002 as 0.561 , and has continuously increased reaching 1.677 for 2014. ANM currently receives more than 400 manuscripts annually. I am happy to share with you the fact that about $75 \%$ of submissions have come from overseas countries in recent years, making me very proud of the aforementioned will of the founding members of ANM.

None of these accomplishments would have been realized without the enthusiastic efforts of numerous contributors including editors, reviewers and authors. As the current editor-in-chief, I would like to express my sincere gratitude to all of our reviewers throughout the world who

Seigo Kinuya

kinuya@med.kanazawa-u.ac.jp

1 Department of Nuclear Medicine, Kanazawa University Graduate School of Medical Science, 13-1 Takaramachi, Kanazawa, Ishikawa 920-8641, Japan spare their valuable time to judge manuscripts. I really recognize that review comments have become more and more valuable for authors than those of 6 years ago when I succeeded as chief. Their comments improve the quality of published articles thereby attracting more potential authors, and submissions of manuscripts of high quality studies. Needless to say, a major factor accounting for this favorable trend is the hard work of the associate editors handling the manuscripts.

ANM has continuously obtained a governmental budget from the Japan Society for the Promotion of Science (JSPS) for many years. In 2012, ANM made a successful bid to win the competition for a 5-year budget project of JSPS from 2013 through 2017, resulting in approximately one hundred million JPY (US\$ 820,000) for 5 years. This budget is designated for the worldwide promotion of scientific journals. To meet this goal, ANM has set several goals such as reconstruction of the editorial board consisting of active international members, increase of associate editors for better handling of manuscripts, and promotion on the occasion of international conferences, many of which have already been accomplished.

From this year, ANM will stop distributing printed copies of the journal to help reduce the cost of publication. By doing this and using the governmental budget, ANM can select a number of papers as open access articles. You may know that open access articles are accessible without any membership or charge, instead of which authors have to pay an expensive article processing fee to make their article open access. In past years, ANM selected about 5 articles as open access per year without charging the authors. From this year, ANM will select more articles of significant importance for open access.

ANM will enthusiastically contribute to the development of nuclear medicine and related fields in the world. 
With this in mind I would like to ask all colleagues to keep their eyes on ANM and to continue to contribute to its success.
Seigo Kinuya, M.D., Ph.D.

Editor-in-Chief

Annals of Nuclear Medicine 